\title{
Biquandle Virtual Brackets
}

\author{
Sam Nelson* $\quad$ Kanako Oshiro ${ }^{\dagger} \quad$ Ayaka Shimizu $^{\ddagger} \quad$ Yoshiro Yaguchi $^{\S}$
}

\begin{abstract}
We introduce an infinite family of quantum enhancements of the biquandle counting invariant we call biquandle virtual brackets. Defined in terms of skein invariants of biquandle colored oriented knot and link diagrams with values in a commutative ring $R$ using virtual crossings as smoothings, these invariants take the form of multisets of elements of $R$ and can be written in a "polynomial" form for convenience. The family of invariants defined herein includes as special cases all quandle and biquandle 2-cocycle invariants, all classical skein invariants (Alexander-Conway, Jones, HOMFLYPT and Kauffman polynomials) and all biquandle bracket invariants defined in 12 as well as new invariants defined using virtual crossings in a fundamental way, without an obvious purely classical definition.
\end{abstract}

KEYwORDS: Quantum enhancements, biquandles, biquandle counting invariants, virtual knots and links

2010 MSC: 57M27, 57M25

\section{Introduction}

In 14 the notion was introduced of quantum enhancements of the biquandle counting invariant by means of quantum invariants of biquandle-colored knots and links. More precisely, if $\beta$ is a quantum invariant of biquandle colored knots and links, then the multiset of $\beta$-values over the set of biquandle colorings of a knot or link $K$ is an invariant whose cardinality recovers the biquandle counting invariant. In [12], a family of such quantum enhancements including both classical skein invariants and biquandle cocycle invariants as special cases, known as biquandle brackets, was introduced. These invariants can be understood as skein invariants of biquandle colored oriented knots and links with skein coefficients depending on the biquandle colors of the semiarcs involved in the crossing being smoothed. The fact that a biquandle coloring is "broken" by smoothings means it is simpler to think of the invariant in terms of the state-sum definition in which all smoothings are done at once rather than smoothing crossings one at a time. In [10, picture-valued biquandle brackets were introduced, and in [13 trace diagrams are used to compute biquandle brackets via a recursive expansion instead of via the state-sum definition.

In this paper we generalize the biquandle bracket idea to use a different set of skein relations in which a virtual crossing is considered as a type of a smoothing rather than a kind of crossing. We use the term "biquandle virtual bracket" to distinguish this case from "virtual biquandle brackets" which are brackets with coefficients in a virtual biquandle. This new infinite family of invariants of oriented classical and virtual knots and links contains classical biquandle brackets (and hence classical skein invariants and biquandle 2-cocycle invariants) as special cases and uses virtual knot theory in a fundamental way, joining the finite type invariants of 8 as a family of invariants of classical knots without an obvious definition in term of purely classical knot and link diagrams.

The paper is organized as follows. In Section 2 we briefly review biquandles, biquandle colorings of oriented classical and virtual knots and links, and the biquandle counting invariant. In Section 3 we introduce

\footnotetext{
*Email: Sam.Nelson@cmc.edu. Partially supported by Simons Foundation collaboration grant 316709

${ }^{\dagger}$ Email: oshirok@sophia.ac.jp. Partially supported by JSPS KAKENHI Grant Number 16K17600.

‡Email: shimizu@nat.gunma-ct.ac.jp. Partially supported by Grant for Basic Science Research Projects from The Sumitomo Foundation (160154).

§Email: yaguchi-y@nat.gunma-ct.ac.jp
} 
biquandle virtual brackets. In Section 4 we provide some applications and examples. In Section 5 we close with some questions for future research.

\section{Biquandles and Virtual Links}

We begin with a definition (see $5[7]$ ).

Definition 1. A biquandle is a set $X$ with operations $\underline{\unrhd}, \bar{\triangleright}: X \rightarrow X$ satisfying for all $x, y, z \in X$

(i) $x \unrhd x=x \bar{\triangleright} x$,

(ii) the maps $\alpha_{x}, \beta_{x}: X \rightarrow X$ and $S: X \times X \rightarrow X \times X$ given by

$$
\alpha_{x}(y)=y \bar{\triangleright} x, \quad \beta_{x}(y)=y \unrhd x \quad \text { and } \quad S(x, y)=(y \bar{\triangleright} x, x \unrhd y)
$$

are invertible, and

(iii) we have the exchange laws

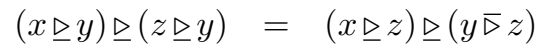

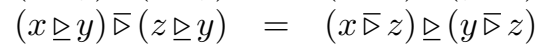

$$
\begin{aligned}
& (x \bar{\triangleright} y) \bar{\nabla}(z \bar{\triangleright} y)=(x \bar{\triangleright} z) \bar{\triangleright}(y \unrhd z) .
\end{aligned}
$$

It is sometimes convenient to write $x \unrhd y$ as $x^{y}$ and $x \bar{\triangleright} y$ as $x_{y}$ for the sake of space.

Definition 2. A map $f: X \rightarrow Y$ between biquandles is a biquandle homomorphism if

$$
f(x \unrhd y)=f(x) \unrhd f(y) \quad \text { and } \quad f(x \triangleright y)=f(x) \triangleright f(y)
$$

for all $x, y \in X$. A subset $S \subset X$ of a biquandle $X$ is a subbiquandle if $S$ is closed under the biquandle operations of $X$, including the inverses of the maps in axiom (ii).

Example 1. A biquandle $X$ in which $x \bar{\triangleright} y=x$ for all $y \in X$ is a quandle. Examples of quandles include

- $n$-fold conjugation quandles: Every group $G$ (or union of conjugacy classes in a group) is a quandle under $x \unrhd y=y^{-n} x y^{n}$ for $n \in \mathbb{Z}$,

- Alexander quandles: Every module $X$ over $\mathbb{Z}\left[t^{ \pm 1}\right]$ is a quandle under $x \unrhd y=t x+(1-t) y$,

- symplectic quandles: Every vector space $V$ over a field $\mathbb{F}$ of characteristic $\neq 2$ with a symplectic form $\langle\rangle:, V \times V \rightarrow \mathbb{F}$ is a quandle under $x \unrhd y=x+\langle x, y\rangle y$.

Example 2. Examples of non-quandle biquandles include

- constant action biquandles: Every set $X$ is a biquandle under $x \bar{\triangleright} y=x \unrhd y=\sigma(x)$ for a bijection $\sigma: X \rightarrow X$

- Alexander biquandles: Every module $X$ over the ring $\mathbb{Z}\left[t^{ \pm 1}, s^{ \pm 1}\right]$ is a biquandle under the operations $x \unrhd y=t x+(s-t) y$ and $x \unrhd y=s x$.

- fundamental biquandle of an oriented link: The set $\mathcal{B}(L)$ of equivalence classes of biquandle words in generators corresponding to semiarcs in a diagram of an oriented link $L$ modulo the congruence generated by the crossing relations

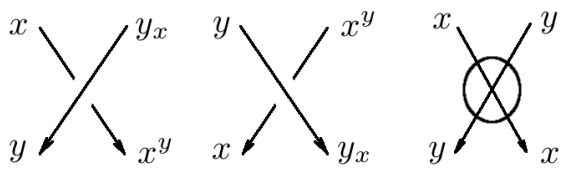


and the biquandle axioms is a strong invariant of virtual links, conjectured to be complete up to reflection for virtual knots and known to be complete up to reflection for classical knots (see [5, 6, 9 for more).

Given a finite set $X=\left\{x_{1}, \ldots, x_{n}\right\}$ we can define a biquandle structure on $X$ by listing operation tables of $\unrhd$ and $\bar{\nabla}$ such that the axioms are satisfied - the diagonals of the tables must agree, the columns must be permutations, etc. We can conveniently encode these tables as an $n \times 2 n$ block matrix by dropping the " $x$ "s and listing only the subscripts, e.g.

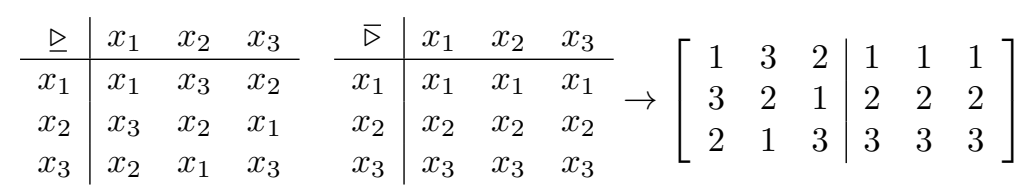

Next, we recall the definition of virtual knots and links (see [1] for more). A virtual knot diagram has classical and virtual crossings as depicted:

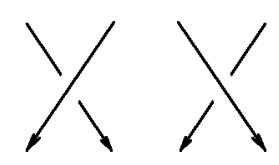

classical

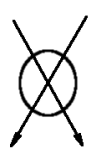

virtual

The virtual crossings are interpreted as not really crossings, but artifacts of drawing a non-planar knot diagram on planar paper - we can regard a virtual crossing as indicating genus in the supporting surface on which the knot diagram is drawn. In this way, a virtual crossing may be considered a kind of smoothing rather than a kind of crossing.

Two virtual link diagrams are equivalent if they are related by the oriented virtual Reidemeister moves:
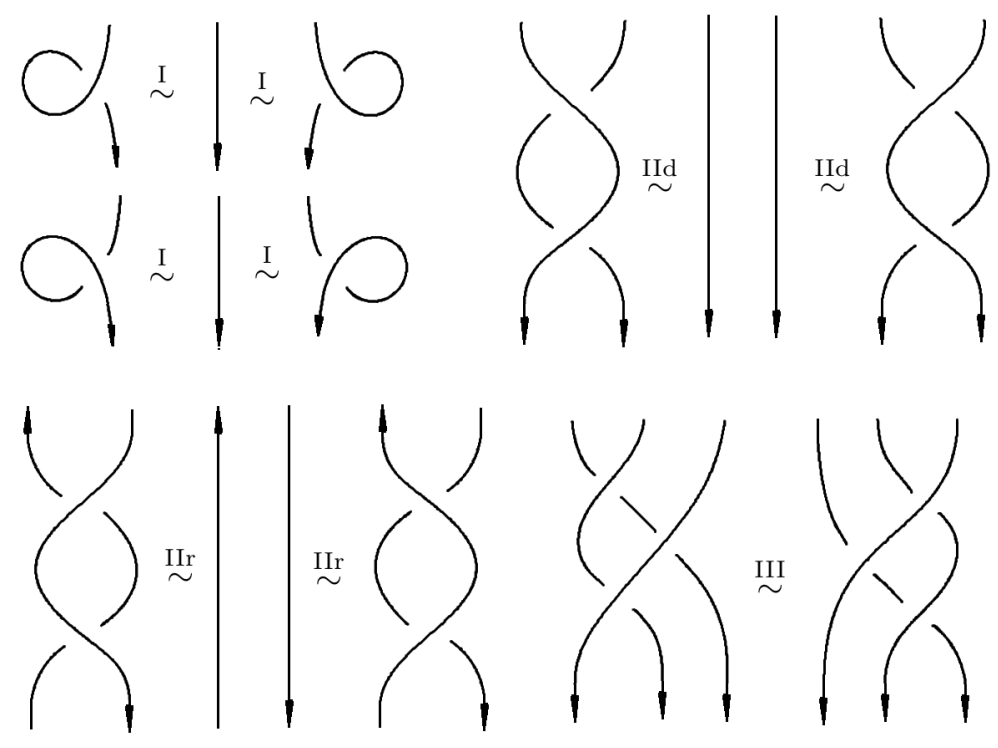


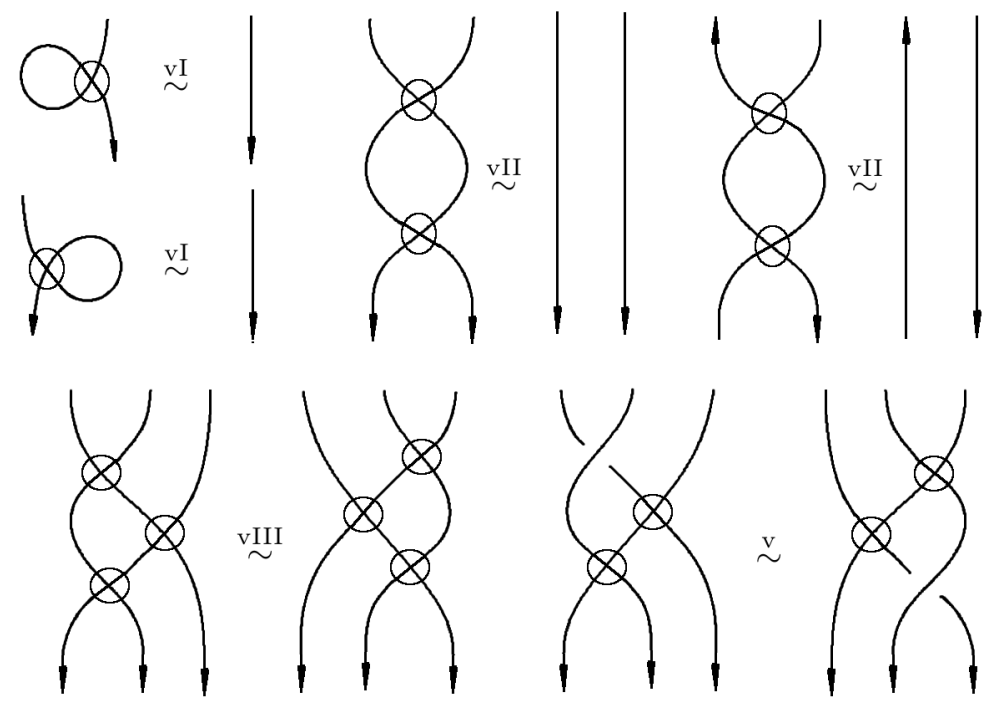

Note that there are more oriented III, vIII and v moves, but in the presence of the other pictured moves they can be recovered from the pictured moves. In particular, the above constitute one generating set of oriented virtual Reidemeister moves. Classical knots and links form a subset of virtual knots and links, with two classical knots or link equivalent as virtual knots or links if and only if they are equivalent as classical knots or links; see [11] for more.

Given a biquandle $X$ and an oriented virtual knot or link $L$ represented by a diagram $D$, we can color $D$ with $X$ by assigning elements of $X$ to the semiarcs in $D$, i.e. the portions of $D$ between crossing points, such that we locally have the following pictures:

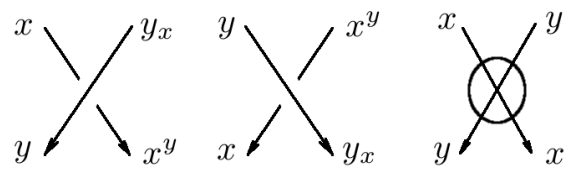

It is then straightforward to verify that for any $X$-coloring of $D$ before a Reidemeister move, there is a unique corresponding coloring after the move. In particular, if $X$ is finite, the number of $X$-colorings of $D$ is finite. In terms of the fundamental biquandle, a coloring is a homomorphism $f: \mathcal{B}(L) \rightarrow X$, and the set of colorings is $\operatorname{Hom}(\mathcal{B}(L), X)$; then the counting invariant is $\Phi_{X}^{\mathbb{Z}}(L)=|\operatorname{Hom}(\mathcal{B}(L), X)|$.

Hence, we have

Theorem 1. The number of $X$-colorings of a virtual knot or link $K$ by a biquandle $X$, denoted $\Phi_{X}^{\mathbb{Z}}(K)$ and known as the biquandle counting invariant, is an invariant of oriented classical and virtual links.

Example 3. The Hopf link below has four colorings by the biquandle $X=\mathbb{Z}_{2}$ with $x \unrhd y=x \bar{\triangleright} y=x+1$ as depicted:
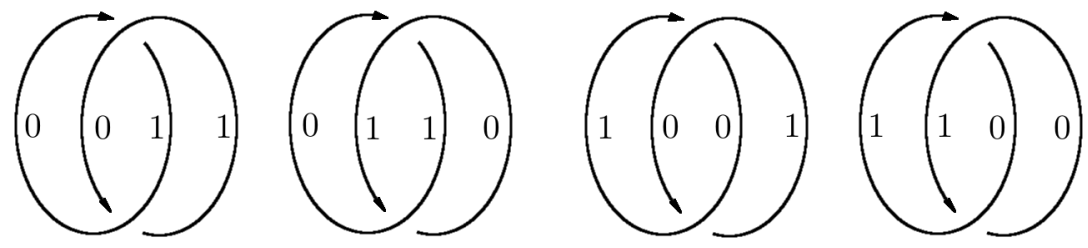
The virtual Hopf link, on the other hand,

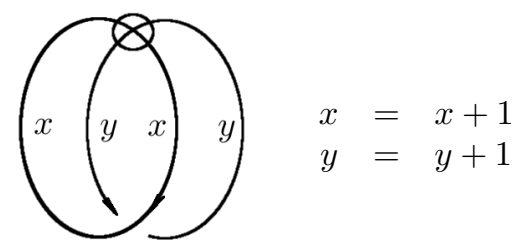

has no colorings by $X$ since there are no solutions to the system of coloring equations, so we have $\Phi_{X}^{\mathbb{Z}}$ (Hopf) $=$ $4 \neq 0=\Phi_{X}^{\mathbb{Z}}(\mathrm{vHopf})$ and the counting invariant distinguishes these two virtual links.

\section{Biquandle Virtual Brackets}

The invariant in Example 3 gives a value of

$$
\Phi_{X}^{\mathbb{Z}}(L)= \begin{cases}2^{c}, & L \notin \mathcal{O} \\ 0, & L \in \mathcal{O}\end{cases}
$$

where $\mathcal{O}$ is the set of virtual links in which any component has an odd number of (classical) crossing points and $c$ is the number of components of $L$. Thus $\Phi_{X}^{\mathbb{Z}}$ with the choice of biquandle $X$ in example 3 fails to distinguish any two classical knots; we would like to strengthen and enhance this and other biquandle counting invariants to obtain stronger invariants. If $\beta$ is an invariant of $X$-colored diagrams, then the multiset of $\beta$-values over the set of $X$-colorings of $L$ is a generally stronger invariant from which we can recover $\Phi_{X}^{\mathbb{Z}}$ by taking the multiset's cardinality. In this section we introduce a new infinite family of such enhancements.

Definition 3. Let $X$ be a biquandle and $R$ a commutative ring with identity. Then a biquandle virtual bracket consists of six maps $A, B, C, D, U, V: X \times X \rightarrow R$ and two distinguished elements $\delta \in R, w \in R^{\times}$ satisfying the following conditions:

$$
\begin{aligned}
& w=\delta A_{x, x}+B_{x, x}+V_{x, x}, \quad(i . i) \\
& w^{-1}=\delta C_{x, x}+D_{x, x}+U_{x, x}, \quad(i . i i), \\
& 1=A_{x, y} C_{x, y}+V_{x, y} U_{x, y}, \quad \text { (ii.i) } \\
& 1=B_{x, y} D_{x, y}+V_{x, y} U_{x, y}, \\
& 0=A_{x, y} U_{x, y}+V_{x, y} C_{x, y}, \quad \text { (ii.iii) } \\
& 0=B_{x, y} U_{x, y}+V_{x, y} D_{x, y}, \quad \text { (ii.iv) } \\
& 0=\delta B_{x, y} D_{x, y}+A_{x, y} D_{x, y}+B_{x, y} C_{x, y}, \quad \text { (ii.v) } \\
& 0=\delta A_{x, y} C_{x, y}+A_{x, y} D_{x, y}+B_{x, y} C_{x, y}, \quad \text { (ii.vi), }
\end{aligned}
$$




$$
\begin{aligned}
& A_{x, y} A_{x^{y}, z_{y}} A_{y, z}+V_{x, y} A_{x^{y}, z_{y}} V_{y, z}=A_{y_{x}, z_{x}} A_{x, z} A_{x^{z}, y^{z}}+V_{y_{x}, z_{x}} A_{x, z} V_{x^{z}, y^{z}} \\
& A_{x, y} A_{x^{y}, z_{y}} B_{y, z}+B_{x, y} A_{x^{y}, z_{y}} A_{y, z} \\
& +\delta B_{x, y} A_{x y}, z_{y} B_{y, z}+B_{x, y} A_{x^{y}, z_{y}} V_{y, z} \\
& +B_{x, y} B_{x^{y}, z_{y}} B_{y, z}+B_{x, y} V_{x^{y}, z_{y}} B_{y, z} \\
& +V_{x, y} A_{x^{y}, z_{y}} B_{y, z}=A_{y_{x}, z_{x}} B_{x, z} A_{x^{z}, y^{z}} \\
& A_{x, y} B_{x^{y}, z_{y}} A_{y, z}=A_{y_{x}, z_{x}} A_{x, z} B_{x^{z}, y^{z}}+B_{y_{x}, z_{x}} A_{x, z} A_{x^{z}, y^{z}} \\
& +\delta B_{y_{x}, z_{x}} A_{x, z} B_{x^{z}, y^{z}}+B_{y_{x}, z_{x}} A_{x, z} V_{x^{z}, y^{z}} \\
& +B_{y_{x}, z_{x}} B_{x, z} B_{x^{z}, y^{z}}+B_{y_{x}, z_{x}} V_{x, z} B_{x^{z}, y^{z}}+V_{y_{x}, z_{x}} A_{x, z} B_{x^{z}, y^{z}} \\
& A_{x, y} V_{x^{y}, z_{y}} A_{y, z}=A_{y_{x}, z_{x}} A_{x, z} V_{x^{z}, y^{z}}+V_{y_{x}, z_{x}} A_{x, z} A_{x^{z}, y^{z}} \\
& A_{x, y} A_{x^{y}, z_{y}} V_{y, z}+V_{x, y} A_{x^{y}, z_{y}} A_{y, z}=A_{y_{x}, z_{x}} V_{x, z} A_{x^{z}, y^{z}} \\
& B_{x, y} B_{x^{y}, z_{y}} A_{y, z}+B_{x, y} V_{x^{y}, z_{y}} V_{y, z}=A_{y_{x}, z_{x}} B_{x, z} B_{x^{z}, y^{z}}+V_{y_{x}, z_{x}} V_{x, z} B_{x^{z}, y^{z}} \\
& A_{x, y} B_{x^{y}, z_{y}} B_{y, z}+V_{x, y} V_{x^{y}, z_{y}} B_{y, z}=B_{y_{x}, z_{x}} B_{x, z} A_{x^{z}, y^{z}}+B_{y_{x}, z_{x}} V_{x, z} V_{x^{z}, y^{z}} \\
& B_{x, y} B_{x^{y}, z_{y}} V_{y, z}+B_{x, y} V_{x^{y}, z_{y}} A_{y, z}=A_{y_{x}, z_{x}} B_{x, z} V_{x^{z}, y^{z}} \\
& A_{x, y} B_{x^{y}, z_{y}} V_{y, z}=B_{y_{x}, z_{x}} B_{x, z} V_{x^{z}, y^{z}}+B_{y_{x}, z_{x}} V_{x, z} A_{x^{z}, y^{z}} \\
& V_{x, y} B_{x^{y}, z_{y}} A_{y, z}=A_{y_{x}, z_{x}} V_{x, z} B_{x^{z}, y^{z}}+V_{y_{x}, z_{x}} B_{x, z} B_{x^{z}, y^{z}} \\
& A_{x, y} V_{x^{y}, z_{y}} B_{y, z}+V_{x, y} B_{x^{y}, z_{y}} B_{y, z}=V_{y_{x}, z_{x}} B_{x, z} A_{x^{z}, y^{z}} \\
& V_{x, y} V_{x^{y}, z_{y}} A_{y, z}=A_{y_{x}, z_{x}} V_{x, z} V_{x^{z}, y^{z}} \\
& A_{x, y} V_{x^{y}, z_{y}} V_{y, z}=V_{y_{x}, z_{x}} V_{x, z} A_{x^{z}, y^{z}} \\
& V_{x, y} B_{x^{y}, z_{y}} V_{y, z}=V_{y_{x}, z_{x}} B_{x, z} V_{x^{z}, y^{z}} \\
& V_{x, y} V_{x^{y}, z_{y}} V_{y, z}=V_{y_{x}, z_{x}} V_{x, z} V_{x^{z}, y^{z}}
\end{aligned}
$$

Note that in the case that $A_{x, y}, B_{x, y}, C_{x, y}$ and $D_{x, y}$ are all invertible for a pair $(x, y)$, conditions (ii.iii) and (ii.iv) reduce to

$$
\begin{aligned}
& \delta=-A_{x, y} B_{x, y}^{-1}-C_{x, y} D_{x, y}^{-1}, \quad\left(i i . i i i^{\prime}\right) \\
& \delta=-A_{x, y}^{-1} B_{x, y}-C_{x, y}^{-1} D_{x, y} \quad\left(i i . i v^{\prime}\right) .
\end{aligned}
$$

The biquandle virtual bracket axioms are chosen so that the skein relations

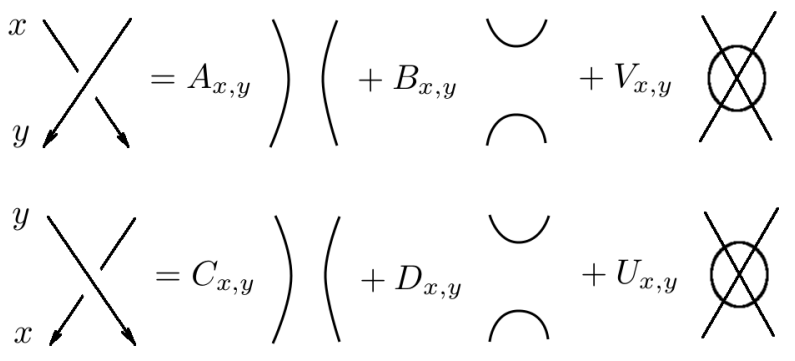

with writhe correction factor $w$ and smoothed component value $\delta$
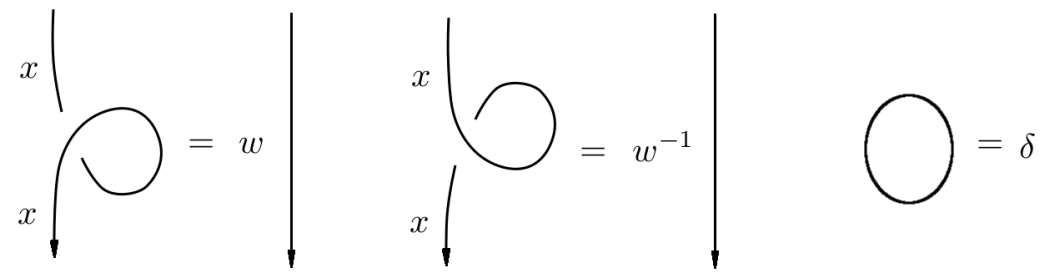

define an invariant of $X$-colored Reidemeister moves.

To accomplish this, we start with the idea that for a given $X$-coloring $f$ of an oriented link diagram $L$ with $c$ classical crossings, there is a set of $3^{c}$ completely smoothed diagrams known as states in which each classical crossing is smoothed either with the orientation, against the orientation, or made virtual. Each such state $S$ has an associated value $\beta_{S} \in R$ defined as the product of the coefficients associated to the choices of 
smoothings times $\delta^{k}$ where $k$ is the number of closed curve components of the state (which may have virtual crossings) times $w^{n-p}$ where $n$ is the number of negative classical crossings and $p$ is the number of positive classical crossings of $L$. We then sum these $\beta_{S}$ values over the complete set of states obtained from $f$ to obtain the state sum value $\beta_{f}$ for the given coloring of the link, and the multiset of $\beta$ values over the set of all $X$-colorings $f$ of $L$ is then an invariant of oriented links.

We have the following critical observation:

Observation 1. A move on a tangle which does not change connectivity on the boundary (i.e., boundary points connected or not connected in the pre-move tangle are still connected or not connected in the post-move tangle) and for which the coefficient weight sums are equal before and after the move does not change the overall contribution to the state sum.

Using this observation, we obtain the biquandle virtual bracket axioms from the oriented virtual Reidemeister moves.

The Reidemeister I moves require that $w=\delta A_{x, x}+B_{x, x}+V_{x, x}$ and $w^{-1}=\delta C_{x, x}+D_{x, x}+U_{x, x}$ :

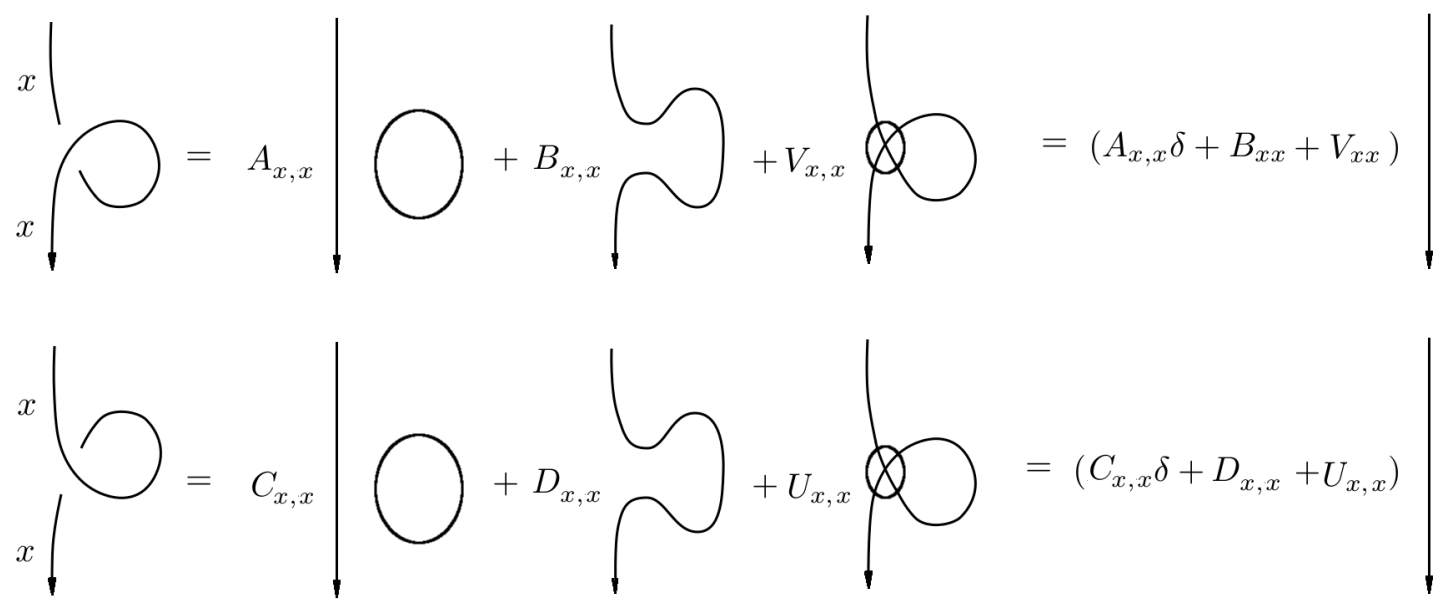

Comparing the skein expansions before and after the direct and reverse oriented Reidemeister II moves yields the conditions

$$
\begin{aligned}
& 1=A_{x, y} C_{x, y}+V_{x, y} U_{x, y} \\
& 1=B_{x, y} D_{x, y}+V_{x, y} U_{x, y} \\
& 0=A_{x, y} U_{x, y}+V_{x, y} C_{x, y} \\
& 0=B_{x, y} U_{x, y}+V_{x, y} D_{x, y} \\
& 0=\delta B_{x, y} D_{x, y}+A_{x, y} D_{x, y}+B_{x, y} C_{x, y} \\
& 0=\delta A_{x, y} C_{x, y}+A_{x, y} D_{x, y}+B_{x, y} C_{x, y}
\end{aligned}
$$




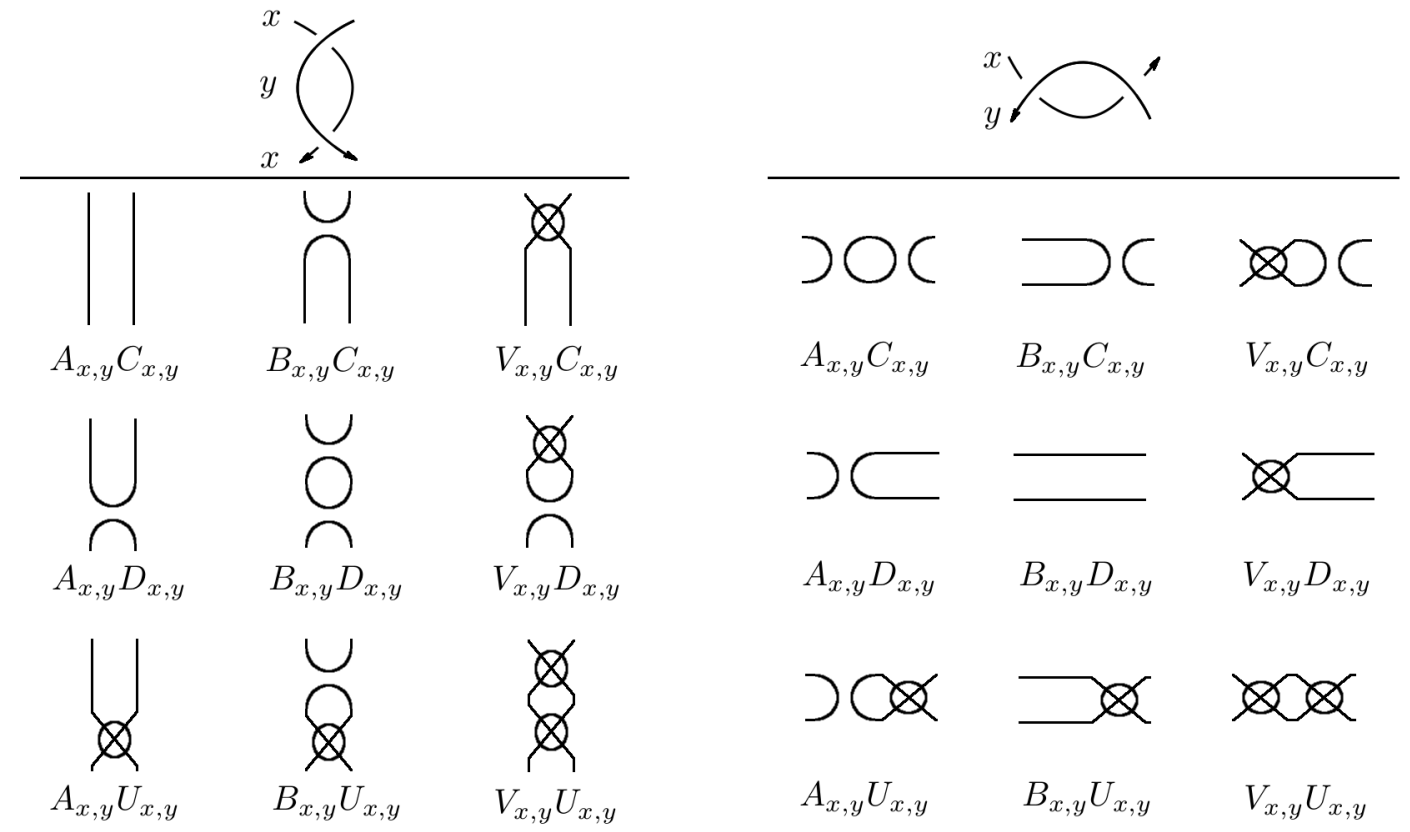

The two sides of the third Reidemeister move, with each smoothed state labeled with its coefficient product,

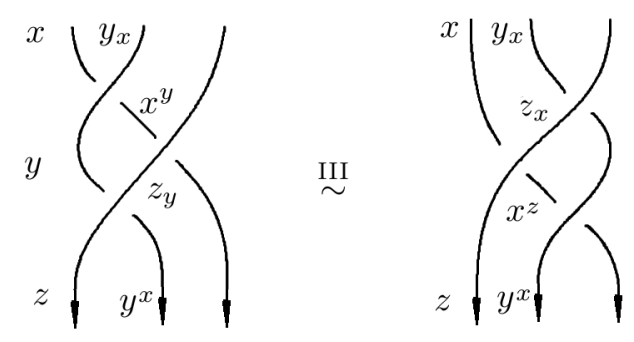


are left-hand side

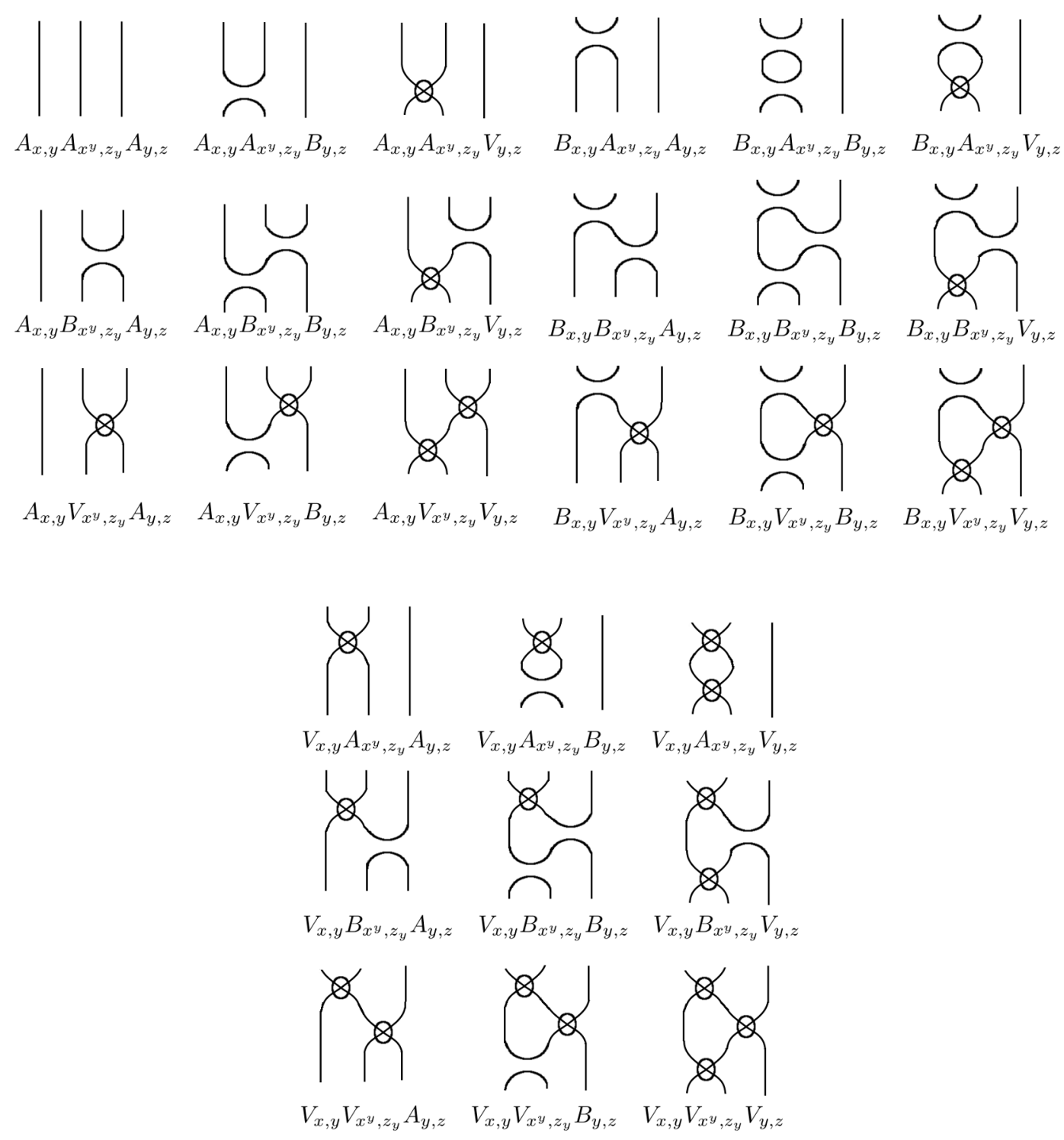


and right-hand side
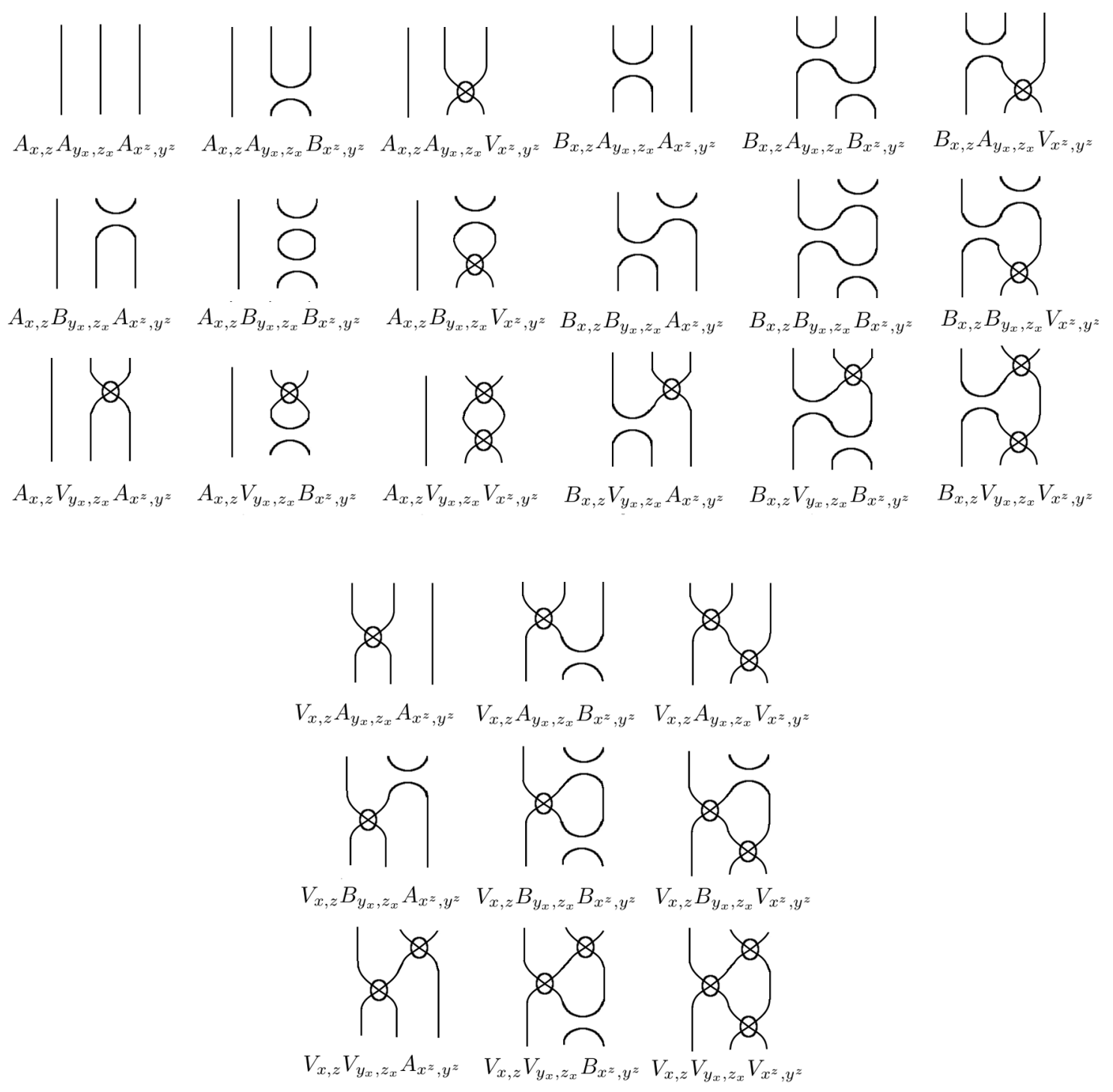

Comparing coefficient sums of equivalent tangles using observation 1 yields the fifteen axioms (iii.i) through (iii.xv).

The pure virtual moves do not affect the state sum value in any way, and the mixed virtual move yields the same sum on both sides up to virtual moves, and thus does not change the state sum:

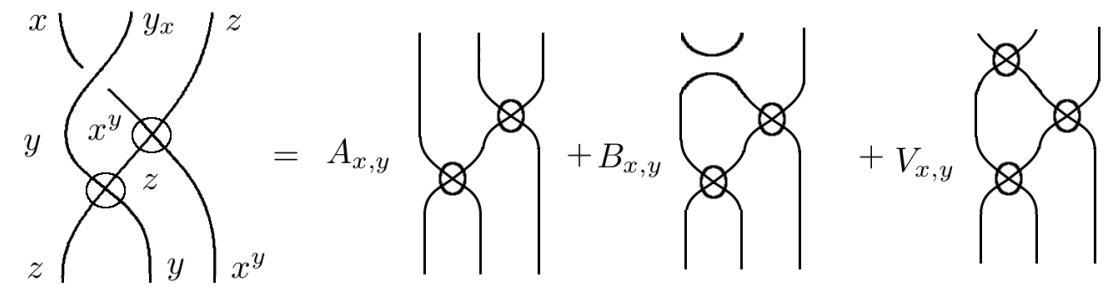




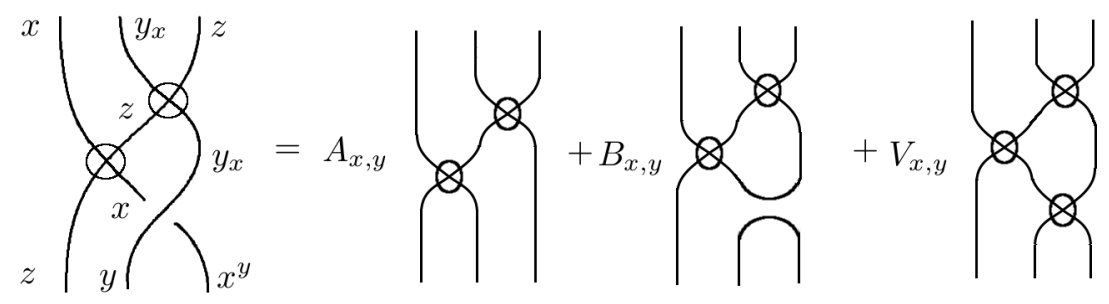

Hence, we have

Theorem 2. Let $X$ be a biquandle and $\beta$ a biquandle virtual bracket. Then for any $X$-coloring $f$ of an oriented (classical or) virtual link $L$, the state sum

$$
\beta_{f}=\sum_{S \text { State }} \beta_{S}
$$

is an invariant of $X$-colored virtual Reidemeister moves.

Example 4. Treating an oriented virtual link $L$ as colored by its fundamental biquandle $\mathcal{B}(L)$ with $f$ assigning to each semiarc its generator, the state sum $\beta_{f}$ is the fundamental biquandle virtual bracket of L. While it is difficult to compare values of the fundamental virtual bracket directly (since doing so requires comparing fundamental biquandles of links directly), we find it useful to use the fundamental virtual bracket expression as a step in computing state sums for virtual brackets over finite biquandles by substituting image values of generators under the coloring maps $f \in \operatorname{Hom}(\mathcal{B}(L), X)$; see Section 4 .

Corollary 3. For any biquandle $X$, biquandle virtual bracket $\beta$ and oriented virtual link L, the multiset of state sum values

$$
\Phi_{X}^{\beta, M}(L)=\left\{\beta_{f} \mid f \in \operatorname{Hom}(\mathcal{B}(L), X)\right\}
$$

is an invariant of classical and virtual links.

Definition 4. We call the multiset in Corollary 3 the biquandle virtual bracket multiset invariant of $L$ with respect to the biquandle $X$ and the biquandle virtual bracket $\beta$.

If $R$ is a number ring, it is common practice (see [3,5] etc.) to write the multiset in a "polynomial" form vaguely analogous to a generating function by making the multiplicities coefficients and the elements exponents, e.g. encoding $\{-2,1,1,1,3,3\}$ as $u^{-2}+3 u+2 u^{3}$. When written this way, we will refer to the invariant as the biquandle virtual bracket polynomial of $L$, denoted $\Phi_{X}^{\beta}(L)$.

Example 5. Let $X$ be a biquandle and $\beta$ a biquandle virtual bracket over a commutative ring with identity $R$. If $V_{x, y}=U_{x, y}=0$ for all $x, y \in X$, then $\beta$ is a biquandle bracket as defined in [12]. We will refer to such virtual brackets as biquandle classical brackets or just biquandle brackets.

Example 6. Let $X$ be a biquandle and $\beta$ a biquandle virtual bracket over a commutative ring with identity $R$. If $A_{x, y}=B_{x, y}=C_{x, y}=D_{x, y}=0$ for all $x, y \in X$ and $w=\delta=1$, then the virtual bracket axioms reduce to

$$
U_{x, y}=V_{x, y}^{-1} \quad \text { and } \quad V_{x, y} V_{x^{y}, z_{y}} V_{y, z}=V_{y_{x}, z_{x}} V_{x, z} V_{x^{z}, y^{z}}
$$

for all $x, y, z \in X$, making $V$ a biquandle 2-cocycle and $\Phi_{X}^{B}$ exactly the biquandle 2-cocycle invariant with Boltzmann weights written multiplicatively (see $2,4,5,5)$. 


\section{Applications and Examples}

In this section, we provide examples which show that our invariants, including the special case of biquandle classical brackets from [12, can be used to determine chirality of a virtual knot or link, or to determine non-invertibility of a virtual knot or link.

Example 7. Let $X=\mathbb{Z}_{2}$ be the biquandle with operations $\unrhd, \bar{\triangleright}: X \times X \rightarrow X$ defined by

$$
x \unrhd y=x+1 \quad \text { and } \quad x \bar{\triangleright} y=x+1 .
$$

Let $\delta=3 \in \mathbb{Z}_{5}$. Define $A_{x y}, B_{x y}, V_{x y}, C_{x y}, D_{x y}, U_{x y}: X \times X \rightarrow \mathbb{Z}_{5}$ by

$$
\left[A_{x y}\left|B_{x y}\right| V_{x y}\left|C_{x y}\right| D_{x y} \mid U_{x y}\right]=\left[\begin{array}{ll|ll|ll|ll|ll|ll}
1 & 1 & 1 & 1 & 0 & 2 & 1 & 3 & 1 & 3 & 0 & 4 \\
1 & 1 & 1 & 1 & 3 & 0 & 3 & 1 & 3 & 1 & 1 & 0
\end{array}\right] .
$$

Our Python and Mathematica computations agree that this data defines a biquandle virtual bracket structure over $X$.

The right-handed virtual trefoil $L_{1}^{\text {vtrefoil }}$ below has nine states with coefficients as listed.
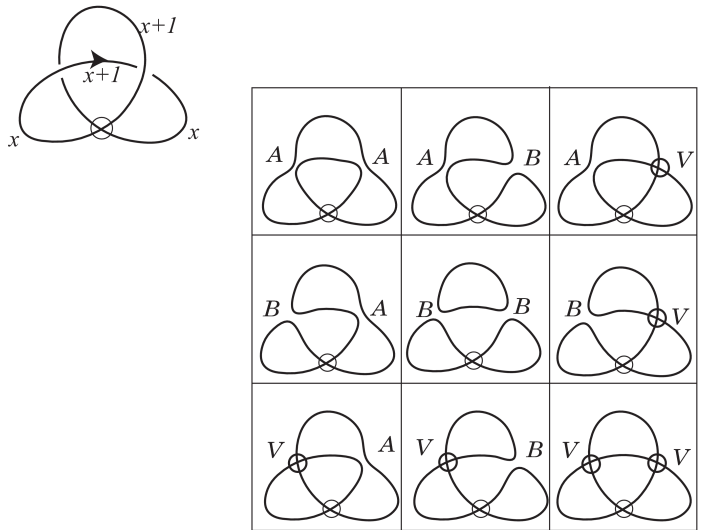

Then the fundamental biquandle virtual bracket value with respect to the biquandle $X$ is

$$
\begin{aligned}
\phi_{1}(x)= & \left(A_{x x+1} V_{x+1 x}+B_{x x+1} B_{x+1 x}+V_{x x+1} A_{x+1 x}\right) \delta^{2} \\
& +\left(A_{x x+1} A_{x+1 x}+A_{x x+1} B_{x+1 x}+B_{x x+1} A_{x+1 x}+B_{x x+1} V_{x+1 x}+V_{x x+1} B_{x+1 x}+V_{x x+1} V_{x+1 x}\right) \delta .
\end{aligned}
$$

Hence we have

$$
\begin{array}{c|c}
x & \phi_{1}(x) \\
\hline 0 & (1 \cdot 3+1 \cdot 1+2 \cdot 1) \cdot 3^{2}+(1 \cdot 1+1 \cdot 1+1 \cdot 1+1 \cdot 3+2 \cdot 1+2 \cdot 3) \cdot 3=1 \\
1 & (1 \cdot 2+1 \cdot 1+3 \cdot 1) \cdot 3^{2}+(1 \cdot 1+1 \cdot 1+1 \cdot 1+1 \cdot 2+3 \cdot 1+3 \cdot 2) \cdot 3=1
\end{array}
$$

Therefore the right-handed virtual trefoil $L_{1}^{\text {vtrefoil }}$ has biquandle virtual bracket invariant

$$
\Phi\left(L_{1}^{\mathrm{vtrefoil}}\right)=2 u \text {. }
$$


The left-handed virtual trefoil $L_{2}^{\text {vtrefoil }}$ below has nine states with coefficients as listed.

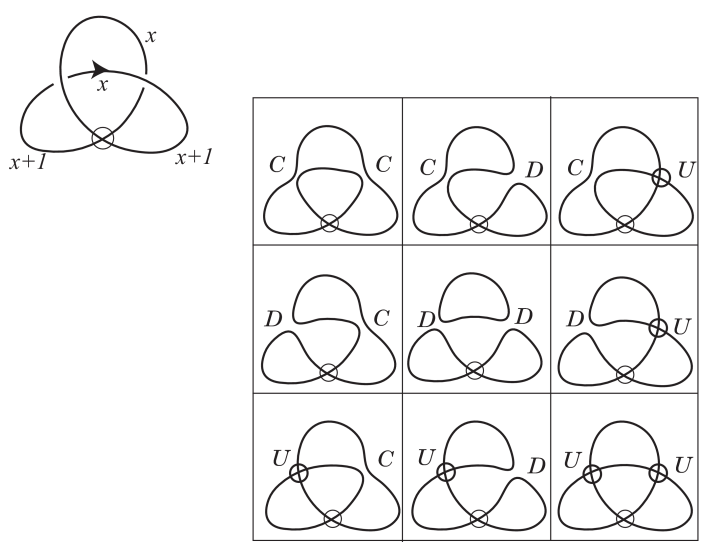

Then the fundamental biquandle virtual bracket value with respect to $X$ is

$$
\begin{aligned}
\phi_{2}(x)= & \left(C_{x x+1} U_{x+1 x}+D_{x x+1} D_{x+1 x}+U_{x x+1} C_{x+1 x}\right) \delta^{2} \\
& +\left(C_{x x+1} C_{x+1 x}+C_{x x+1} D_{x+1 x}+D_{x x+1} C_{x+1 x}+D_{x x+1} U_{x+1 x}+U_{x x+1} D_{x+1 x}+U_{x x+1} U_{x+1 x}\right) \delta .
\end{aligned}
$$

Hence we have

$$
\begin{array}{c|c}
x & \phi_{2}(x) \\
\hline 0 & (3 \cdot 1+3 \cdot 3+4 \cdot 3) \cdot 3^{2}+(3 \cdot 3+3 \cdot 3+3 \cdot 3+3 \cdot 1+4 \cdot 3+4 \cdot 1) \cdot 3=4 \\
1 & (3 \cdot 4+3 \cdot 3+1 \cdot 3) \cdot 3^{2}+(3 \cdot 3+3 \cdot 3+3 \cdot 3+3 \cdot 4+1 \cdot 3+1 \cdot 4) \cdot 3=4
\end{array}
$$

Therefore the left-handed virtual trefoil $L_{2}^{\text {vtrefoil }}$ has biquandle virtual bracket invariant

$$
\Phi\left(L_{2}^{\text {vtrefoil }}\right)=2 u^{4} .
$$

Therefore the biquandle virtual bracket invariants of the right- and left-handed virtual trefoils (with any orientation) have the different values, and thus, they are not equivalent. Note that it is known that the right-handed (or left-handed) virtual trefoil is equivalent to its inverse.

Example 8. Let $X=\mathbb{Z}_{3}$ be the biquandle with operations $\unrhd, \bar{\triangleright}: X \times X \rightarrow X$ defined by

$$
x \unrhd y=x+2 \quad \text { and } \quad x \bar{\triangleright} y=x+2 .
$$

Let $\delta=2 \in \mathbb{Z}_{3}$. Define $A_{x y}, B_{x y}, V_{x y}, C_{x y}, D_{x y}, U_{x y}: X \times X \rightarrow \mathbb{Z}_{3}$ by

$$
\begin{aligned}
& {\left[A_{x y}\left|B_{x y}\right| V_{x y}\left|C_{x y}\right| D_{x y} \mid U_{x y}\right]} \\
& =\left[\begin{array}{lll|lll|lll|lll|lll|lll}
1 & 1 & 2 & 2 & 2 & 1 & 0 & 0 & 0 & 1 & 1 & 2 & 2 & 2 & 1 & 0 & 0 & 0 \\
2 & 1 & 2 & 1 & 2 & 1 & 0 & 0 & 0 & 2 & 1 & 2 & 1 & 2 & 1 & 0 & 0 & 0 \\
1 & 1 & 1 & 2 & 2 & 2 & 0 & 0 & 0 & 1 & 1 & 1 & 2 & 2 & 2 & 0 & 0 & 0
\end{array}\right] .
\end{aligned}
$$

Our Python and Mathematica computations agree that this defines a biquandle virtual bracket structure.

Let $K_{1}$ be the oriented virtual-knot depicted below.

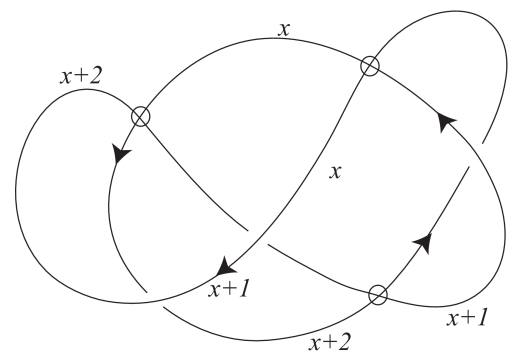


$K_{1}$ has twenty-seven states and its fundamental biquandle virtual bracket value with respect to the biquandle $X$ is

$$
\begin{aligned}
& \phi_{1}(x)=V_{x x+2} A_{x+2 x+1} C_{x x+1} \delta^{3} \\
& +\left(A_{x x+2} A_{x+2 x+1} C_{x x+1}+A_{x x+2} B_{x+2 x+1} D_{x x+1}\right. \\
& +A_{x x+2} V_{x+2 x+1} U_{x x+1}+B_{x x+2} A_{x+2 x+1} C_{x x+1} \\
& +B_{x x+2} B_{x+2 x+1} U_{x x+1}+B_{x x+2} V_{x+2 x+1} D_{x x+1} \\
& +V_{x x+2} A_{x+2 x+1} D_{x x+1}+V_{x x+2} A_{x+2 x+1} U_{x x+1} \\
& \left.+V_{x x+2} B_{x+2 x+1} C_{x x+1}+V_{x x+2} V_{x+2 x+1} C_{x x+1}\right) \delta^{2} \\
& +\left(A_{x x+2} A_{x+2 x+1} D_{x x+1}+A_{x x+2} A_{x+2 x+1} U_{x x+1}\right. \\
& +A_{x x+2} B_{x+2 x+1} C_{x x+1}+A_{x x+2} B_{x+2 x+1} U_{x x+1} \\
& +A_{x x+2} V_{x+2 x+1} C_{x x+1}+A_{x x+2} V_{x+2 x+1} D_{x x+1} \\
& +B_{x x+2} A_{x+2 x+1} D_{x x+1}+B_{x x+2} A_{x+2 x+1} U_{x x+1} \\
& +B_{x x+2} B_{x+2 x+1} C_{x x+1}+B_{x x+2} B_{x+2 x+1} D_{x x+1} \\
& +B_{x x+2} V_{x+2 x+1} C_{x x+1}+B_{x x+2} V_{x+2 x+1} U_{x x+1} \\
& +V_{x x+2} B_{x+2 x+1} D_{x x+1}+V_{x x+2} B_{x+2 x+1} U_{x x+1} \\
& \left.+V_{x x+2} V_{x+2 x+1} D_{x x+1}+V_{x x+2} V_{x+2 x+1} U_{x x+1}\right) \delta,
\end{aligned}
$$

and we can check that $\phi_{1}(0)=\phi_{1}(1)=\phi_{1}(2)=1$. Therefore $K_{1}$ has biquandle virtual bracket invariant

$$
\Phi\left(K_{1}\right)=3 u \text {. }
$$

Let $K_{2}$ be the oriented virtual-knot depicted below, that is, it is the same virtual-knot $K_{1}$ with its orientation reversed.

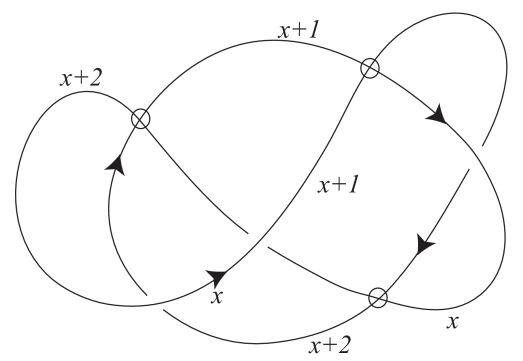

$K_{2}$ has twenty-seven states and its fundamental biquandle virtual bracket value with respect to the biquandle $X$ is

$$
\begin{aligned}
& \phi_{2}(x)=V_{x+2 x} A_{x x+1} C_{x+2 x+1} \delta^{3} \\
& +\left(A_{x+2 x} A_{x x+1} C_{x+2 x+1}+A_{x+2 x} B_{x x+1} D_{x+2 x+1}\right. \\
& +A_{x+2 x} V_{x x+1} U_{x+2 x+1}+B_{x+2 x} A_{x x+1} C_{x+2 x+1} \\
& +B_{x+2 x} B_{x x+1} U_{x+2 x+1}+B_{x+2 x} V_{x x+1} D_{x+2 x+1} \\
& +V_{x+2} A_{x x+1} D_{x+2 x+1}+V_{x+2} A_{x x+1} U_{x+2 x+1} \\
& \left.+V_{x+2 x} B_{x x+1} C_{x+2 x+1}+V_{x+2 x} V_{x x+1} C_{x+2 x+1}\right) \delta^{2} \\
& +\left(A_{x+2}{ }_{x} A_{x x+1} D_{x+2 x+1}+A_{x+2 x} A_{x x+1} U_{x+2 x+1}\right. \\
& +A_{x+2 x} B_{x x+1} C_{x+2 x+1}+A_{x+2} B_{x x+1} U_{x+2 x+1} \\
& +A_{x+2} V_{x x+1} C_{x+2 x+1}+A_{x+2} V_{x x+1} D_{x+2 x+1} \\
& +B_{x+2 x} A_{x x+1} D_{x+2 x+1}+B_{x+2} A_{x x+1} U_{x+2 x+1} \\
& +B_{x+2 x} B_{x x+1} C_{x+2 x+1}+B_{x+2 x} B_{x x+1} D_{x+2 x+1} \\
& +B_{x+2} V_{x x+1} C_{x+2 x+1}+B_{x+2} V_{x x+1} U_{x+2 x+1} \\
& +V_{x+2} B_{x x+1} D_{x+2 x+1}+V_{x+2 x} B_{x x+1} U_{x+2 x+1} \\
& \left.+V_{x+2 x} V_{x x+1} D_{x+2 x+1}+V_{x+2} V_{x x+1} U_{x+2 x+1}\right) \delta,
\end{aligned}
$$

and we can check that $\phi_{1}(0)=\phi_{1}(1)=\phi_{1}(2)=2$. Therefore $K_{2}$ has biquandle bracket invariant

$$
\Phi\left(K_{2}\right)=3 u^{2} .
$$

Therefore the biquandle virtual bracket invariants of $K_{1}$ and $K_{2}=-K_{1}$ have the different values, and thus, they are not equivalent. In particular, $\Phi_{X}^{B}$ can detect invertibility. 
Example 9. Let $X=\mathbb{Z}_{2}$ be the biquandle with operations $\unrhd, \bar{\triangleright}: X \times X \rightarrow X$ defined by

$$
x \unrhd y=x+1 \quad \text { and } \quad x \bar{\nabla}=x+1 .
$$

Let $\delta=3 \in \mathbb{Z}_{5}$. Define $A_{x y}, B_{x y}, V_{x y}, C_{x y}, D_{x y}, U_{x y}: X \times X \rightarrow \mathbb{Z}_{5}$ by

$$
\left[A_{x y}\left|B_{x y}\right| V_{x y}\left|C_{x y}\right| D_{x y} \mid U_{x y}\right]=\left[\begin{array}{ll|ll|ll|ll|ll|ll}
1 & 1 & 1 & 1 & 0 & 2 & 1 & 3 & 1 & 3 & 0 & 4 \\
1 & 1 & 1 & 1 & 3 & 0 & 3 & 1 & 3 & 1 & 1 & 0
\end{array}\right] .
$$

Note that these are the same biquandle and biquandle virtual bracket shown in Example 7.

The right-handed Hopf link $L_{1}^{\text {Hopf }}$ below has nine states with coefficients as listed. Then it has the

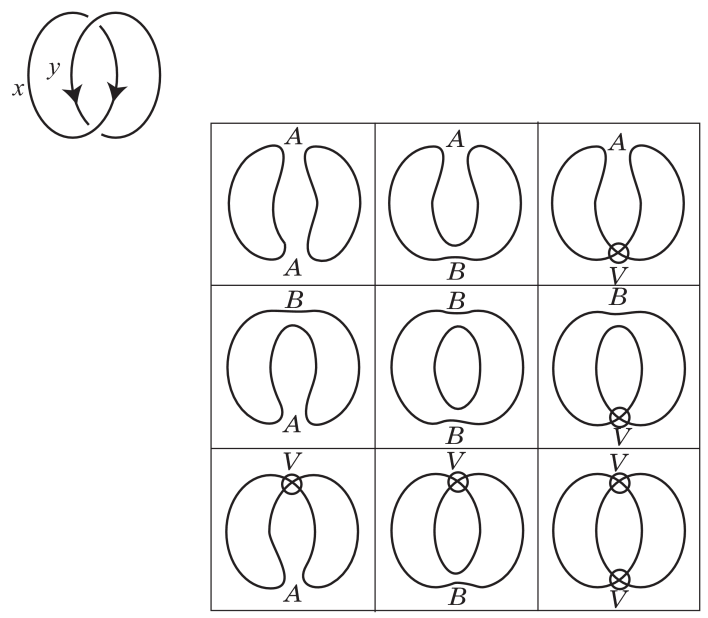

fundamental biquandle virtual bracket value

$$
\begin{aligned}
\phi_{1}(x, y)= & \left(A_{x y} A_{y x}+B_{x y} B_{y x}+V_{x y} V_{y x}\right) \delta^{2} \\
& +\left(A_{x y} B_{y x}+A_{x y} V_{y x}+B_{x y} A_{y x}+B_{x y} V_{y x}+V_{x y} A_{y x}+V_{x y} B_{y x}\right) \delta .
\end{aligned}
$$

Hence we have

$$
\begin{array}{cc|c}
x & y & \phi_{1}(x, y) \\
\hline 0 & 0 & (1 \cdot 1+1 \cdot 1+0 \cdot 0) \cdot 3^{2}+(1 \cdot 1+1 \cdot 0+1 \cdot 1+1 \cdot 0+0 \cdot 1+0 \cdot 1) \cdot 3=4 \\
0 & 1 & (1 \cdot 1+1 \cdot 1+2 \cdot 3) \cdot 3^{2}+(1 \cdot 1+1 \cdot 3+1 \cdot 1+1 \cdot 3+2 \cdot 1+2 \cdot 1) \cdot 3=3 \\
1 & 0 & (1 \cdot 1+1 \cdot 1+3 \cdot 2) \cdot 3^{2}+(1 \cdot 1+1 \cdot 2+1 \cdot 1+1 \cdot 2+3 \cdot 1+3 \cdot 1) \cdot 3=3 \\
1 & 1 & (1 \cdot 1+1 \cdot 1+0 \cdot 0) \cdot 3^{2}+(1 \cdot 1+1 \cdot 0+1 \cdot 1+1 \cdot 0+0 \cdot 1+0 \cdot 1) \cdot 3=4
\end{array}
$$

Therefore the right-handed Hopf link $L_{1}^{\text {Hopf }}$ has biquandle virtual bracket invariant

$$
\Phi\left(L_{1}^{\text {Hopf }}\right)=2 u^{3}+2 u^{4} .
$$

The left-handed Hopf link $L_{2}^{\text {Hopf }}$ below has nine states with coefficients as listed. Then it has the fundamental biquandle virtual bracket value

$$
\begin{aligned}
\phi_{2}(x, y)= & \left(C_{x y} C_{y x}+D_{x y} D_{y x}+U_{x y} U_{y x}\right) \delta^{2} \\
& +\left(C_{x y} D_{y x}+C_{x y} U_{y x}+D_{x y} C_{y x}+D_{x y} U_{y x}+U_{x y} C_{y x}+U_{x y} D_{y x}\right) \delta .
\end{aligned}
$$

Hence we have

$$
\begin{array}{cc|c}
x & y & \phi_{2}(x, y) \\
\hline 0 & 0 & (1 \cdot 1+1 \cdot 1+0 \cdot 0) \cdot 3^{2}+(1 \cdot 1+1 \cdot 0+1 \cdot 1+1 \cdot 0+0 \cdot 1+0 \cdot 1) \cdot 3=4 \\
0 & 1 & (3 \cdot 3+3 \cdot 3+4 \cdot 1) \cdot 3^{2}+(3 \cdot 3+3 \cdot 1+3 \cdot 3+3 \cdot 1+4 \cdot 3+4 \cdot 3) \cdot 3=2 \\
1 & 0 & (3 \cdot 3+3 \cdot 3+1 \cdot 4) \cdot 3^{2}+(3 \cdot 3+3 \cdot 4+3 \cdot 3+3 \cdot 4+1 \cdot 3+1 \cdot 3) \cdot 3=2 \\
1 & 1 & (1 \cdot 1+1 \cdot 1+0 \cdot 0) \cdot 3^{2}+(1 \cdot 1+1 \cdot 0+1 \cdot 1+1 \cdot 0+0 \cdot 1+0 \cdot 1) \cdot 3=4
\end{array}
$$




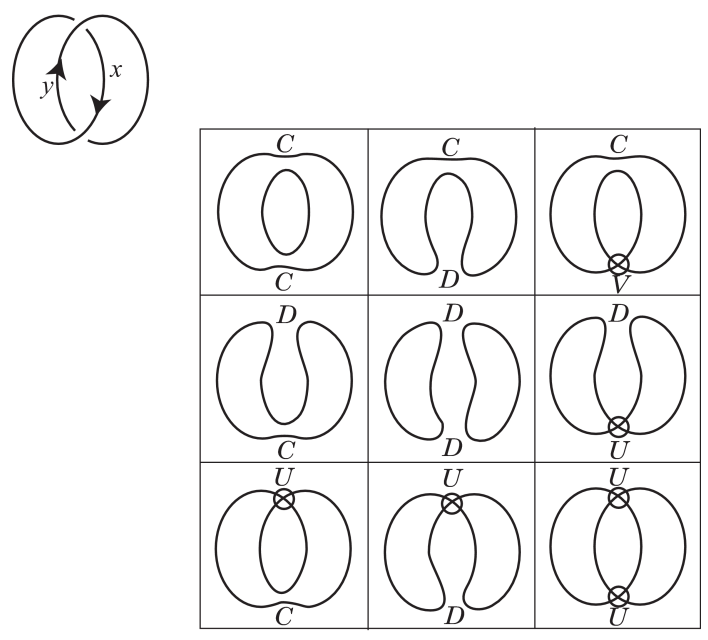

Therefore the left-handed Hopf link $L_{2}^{\text {Hopf }}$ has biquandle bracket invariant

$$
\Phi\left(L_{2}^{\text {Hopf }}\right)=2 u^{2}+2 u^{4} .
$$

On the other hand, the unlink of two components $U_{2}$ has invariant value

$$
\Phi\left(U_{2}\right)=4 u^{4},
$$

and thus, our invariant shows that the right- and left-handed Hopf links are non-trivial. Moreover, the biquandle virtual bracket invariants of the right- and left-handed Hopf links have the different values, which implies that they are not equivalent. In particular, this example shows that some biquandle virtual bracket invariants are sensitive to orientation reversal.

Example 10. Continuing with the same biquandle $X$ from Example 9 , let $R=\mathbb{F}_{8}$, the field of eight elements. Recall that $\mathbb{F}_{8}$ can be written as $\mathbb{Z}_{2}[t] /\left\langle 1+t+t^{3}\right\rangle=\left\{0,1, t, 1+t, t^{2}, 1+t^{2}, t+t^{2}, 1+t+t^{2}\right\}$. Then our Python computations say that the following defines a biquandle virtual bracket over $R$ : Let $\delta=1+t$ and define $A_{x y}, B_{x y}, V_{x y}, C_{x y}, D_{x y}, U_{x y}: X \times X \rightarrow \mathbb{F}_{8}$ by

$$
\begin{aligned}
& {\left[A_{x y}\left|B_{x y}\right| V_{x y}\left|C_{x y}\right| D_{x y} \mid U_{x y}\right]} \\
& =\left[\begin{array}{cc|cc|cc|cc|cc|cc}
1 & 0 & t^{2} & 0 & 0 & t^{2} & 1 & 0 & 1+t+t^{2} & 0 & 0 & 1+t+t^{2} \\
0 & 1 & 0 & t^{2} & 1+t & 0 & 0 & 1 & 0 & 1+t+t^{2} & t+t^{2} & 0
\end{array}\right] .
\end{aligned}
$$

Then our Python computations give the following values for $\Phi_{X}^{\beta, M}(L)$ for prime classical knots with up to eight crossings:

\begin{tabular}{l|r}
$\Phi_{X}^{\beta, M}(L)$ & $L$ \\
\hline$\{2 \times 1\}$ & $5_{2}, 7_{2}, 8_{10}, 8_{11}, 8_{13}, 8_{17}$ \\
$\{2 \times t\}$ & $8_{3}, 8_{6}, 8_{12}, 8_{116}, 8_{18}$ \\
$\{2 \times 1+t\}$ & $U_{1}, 5_{1}, 7_{6}, 8_{15}$ \\
$\left\{2 \times t^{2}\right\}$ & $3_{1}, 6_{2}, 8_{9}$ \\
$\left\{2 \times 1+t^{2}\right\}$ & $4_{1}, 7_{1}, 7_{4}, 8_{5}, 8_{14}$ \\
$\left\{2 \times t+t^{2}\right\}$ & $6_{1}, 6_{3}, 7_{2}, 7_{3}, 8_{7}, 8_{21}$ \\
$\left\{2 \times 1+t+t^{2}\right\}$ & $7_{7}, 8_{2}, 8_{3}, 8_{4}, 8_{8}, 8_{19}, 8_{20}$,
\end{tabular}


for classical links with up to seven crossings:

\begin{tabular}{l|r}
$\Phi_{X}^{\beta, M}(L)$ & $L$ \\
\hline$\{4 \times t\}$ & $L 2 a 1$ \\
$\left\{4 \times 1+t^{2}\right\}$ & $U_{2}$ \\
$\{2 \times 0,2 \times t\}$ & $L 7 a 3$ \\
$\left\{2 \times 0,2 \times 1+t+t^{2}\right\}$ & $L 7 n 2$ \\
$\left\{2 \times 1,2 \times t^{2}\right\}$ & $L 6 a 1$ \\
$\{2 \times t, 2 \times 1+t\}$ & $L 7 a 5$ \\
$\left\{2 \times t, 2 \times 1+t^{2}\right\}$ & $L 7 a 1, L 7 a 4$ \\
$\left\{2 \times t, 2 \times t+t^{2}\right\}$ & $L 7 n 1$ \\
$\left\{2 \times t, 2 \times 1+t+t^{2}\right\}$ & $L 7 a 6$ \\
$\left\{2 \times 1+t, 2 \times t^{2}\right\}$ & $L 7 a 2$ \\
$\left\{2 \times 1+t, 2 \times 1+t+t^{2}\right\}$ & $L 4 a 1, L 6 a 3$, \\
$\left\{2 \times t^{2}, 2 \times t+t^{2}\right\}$ & $L 5 a 1$ \\
$\left\{2 \times t^{2}, 2 \times 1+t+t^{2}\right\}$ & $L 6 a 2$ \\
$\left\{8 \times t^{2}\right\}$ & $U_{3}$ \\
$\{2 \times 1,6 \times t\}$, & $L 6 n 1$ \\
$\left\{2 \times 1+t+t^{2}, 6 \times 1+t^{2}\right\}$ & $L 6 a 4$ \\
$\left\{2 \times t+t^{2}, 6 \times t\right\}$ & $L 6 a 5$ \\
$\{2 \times t, 6 \times 0\}$ & $L 7 a 7$ \\
$\left\{2 \times t+t^{2}, 6 \times 0\right\}$ & $L 6 a 5$, \\
$\left\{2 \times t, 2 \times 1+t, 4 \times t+t^{2}\right\}$ & $L 7 a 7$,
\end{tabular}

and for virtual knots with up to four classical crossings as listed in [1]:

\begin{tabular}{l|r}
$\Phi_{X}^{\beta, M}(L)$ & \multicolumn{1}{c}{$L$} \\
\hline$\{2 \times 0\}$ & $3.5,4.14,4.20,4.21,4.22,4.24,4.34,4.36,4.40,4.52,4.60,4.64,4.68,4.89,4.105$ \\
\hline$\{2 \times t\}$ & $3.2,3.3,3.4,4.4,4.10,4.11,4.16,4.18,4.23,4.27,4.30,4.31,4.33,4.38,4.39,4.41$, \\
& $4.44,4.45,4.49,4.50,4.54,4.57,4.62,4.63,4.65,4.70,4.74,4.79,4.81,4.82,4.83$, \\
& $4.87,4.92,4.95,4.101$ \\
\hline$\{2 \times 1+t\}$ & $U_{1}, 3.1,3.7,4.2,4.6,4.8,4.12,4.13,4.17,4.19,4.26,4.32,4.35,4.42,4.46,4.47,4.51$, \\
& $4.55,4.56,4.58,4.59,4.66,4.67,4.71,4.72,4.75,4.76,4.77,4.85,4.93,4.96,4.97$, \\
& $4.98,4.102,4.103,4.106,4.107$ \\
\hline$\left\{2 \times t^{2}\right\}$ & $4.1,4.3,4.7,4.9,4.15,4.25,4.29,4.37,4.43,4.48,4.53,4.61,4.69,4.73,4.78,4.80$, \\
$\left\{2 \times 1+t^{2}\right\}$ & $4.86,4.90,4.91,4.99,4.100,4.108$ \\
\hline$\left\{2 \times 1+t+t^{2}\right\}$ & $2.1,4.28,4.84,4.88,4.104$.
\end{tabular}

Moreover, $\Phi_{X}^{\beta}$ distinguishes the square knot $3_{1} \# \overline{3_{1}}$ from the positive granny knot $3_{1} \# 3_{1}$, though curiously does not distinguish the square knot from the negative granny knot $\overline{3_{1}} \# \overline{3_{1}}$
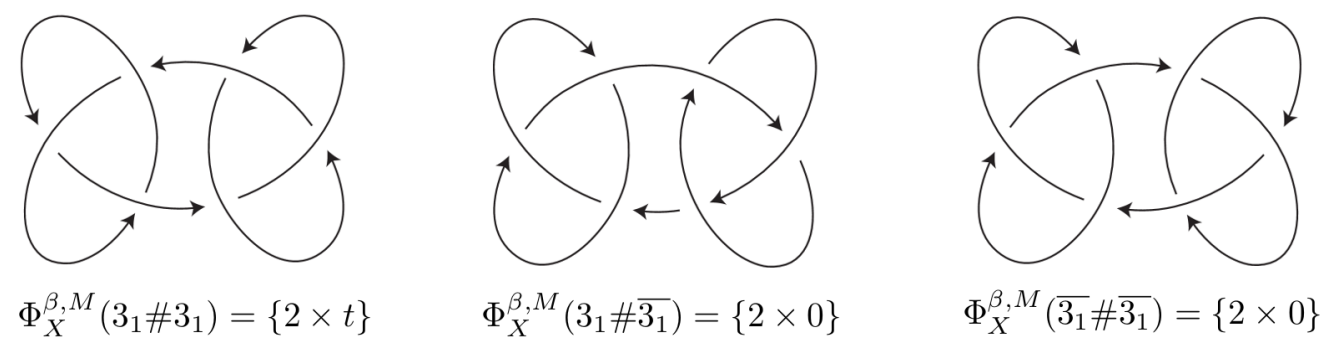

We note that $\Phi_{X}^{\beta}\left(\overline{3_{1}}\right)=\{2 \times 0\}$. 


\section{Questions}

We conclude with some questions for future research.

The case of the square and granny knots example in the last section suggests that at least for some virtual brackets, the $\beta$ values might be multiplicative over connected sum; we ask, for which biquandle virtual brackets $(X, R, \beta)$ do we have $\Phi_{X}^{\beta}\left(K \# K^{\prime}\right)=\Phi_{X}^{\beta}(K) \Phi_{X}^{\beta}\left(K^{\prime}\right)$ ?

We have only considered the cases of extremely small biquandles and coefficient rings using computer search; efficient methods for finding virtual brackets over larger biquandles and coefficient rings are of great interest and should produce strong invariants.

As with any invariant, it is worth asking what kinds of categorifications are possible for these invariants. For Khovanov homology generalizations, it seems perhaps best to start with biquandle virtual brackets over polynomial rings.

\section{References}

[1] D. Bar-Natan. The knot atlas http://katlas.org/wiki/Main_Page.

[2] J. S. Carter, M. Elhamdadi, and M. Saito. Homology theory for the set-theoretic Yang-Baxter equation and knot invariants from generalizations of quandles. Fund. Math., 184:31-54, 2004.

[3] J. S. Carter, D. Jelsovsky, S. Kamada, L. Langford, and M. Saito. State-sum invariants of knotted curves and surfaces from quandle cohomology. Electron. Res. Announc. Amer. Math. Soc., 5:146-156 (electronic), 1999.

[4] J. Ceniceros, M. Elhamdadi, M. Green, and S. Nelson. Augmented biracks and their homology. Internat. J. Math., 25(9):1450087, 19, 2014.

[5] M. Elhamdadi and S. Nelson. Quandles - an introduction to the algebra of knots, volume 74 of Student Mathematical Library. American Mathematical Society, Providence, RI, 2015.

[6] R. Fenn, M. Jordan-Santana, and L. Kauffman. Biquandles and virtual links. Topology Appl., 145(13):157-175, 2004.

[7] R. Fenn, C. Rourke, and B. Sanderson. Trunks and classifying spaces. Appl. Categ. Structures, 3(4):321$356,1995$.

[8] M. Goussarov, M. Polyak, and O. Viro. Finite-type invariants of classical and virtual knots. Topology, 39(5):1045-1068, 2000.

[9] D. Hrencecin and L. H. Kauffman. Biquandles for virtual knots. J. Knot Theory Ramifications, 16(10):1361-1382, 2007.

[10] D. Ilyutko and V. O. Manturov. Picture-valued biquandle bracket. arXiv:math.GT/1701.06011.

[11] L. H. Kauffman. Virtual knot theory. European J. Combin., 20(7):663-690, 1999.

[12] S. Nelson, M. E. Orrison, and V. Rivera. Quantum enhancements and biquandle brackets. J. Knot Theory Ramifications, 26(5):1750034, 24, 2017.

[13] S. Nelson and N. Oyamaguchi. Trace diagrams and biquandle brackets. Internat. J. Math., 28(14):1750104, 24, 2017.

[14] S. Nelson and V. Rivera. Quantum enhancements of involutory birack counting invariants. J. Knot Theory Ramifications, 23(7):1460006, 15, 2014. 
Department of Mathematical Sciences

Claremont McKenna College

850 Columbia Ave.

Claremont, CA 91711

Department of Information and Communication Sciences

SOPHIA UNIVERSITY

7-1 Kiol-Cho, Chiyoda-ku, TOKYo 102-8554, Japan.

Department of Mathematics

National Institute of Technology, Gunma College

580 Toriba-CHO, MAEBASHI-SHI

GUNMA 371-8530, JAPAN. 